\title{
The education and practice program for medical students with quantitative and qualitative fit test for respiratory protective equipment
}

\author{
Jun-Pyo MYONG ${ }^{1}$, JunSu BYUN ${ }^{1}$, YounMo CHO ${ }^{1}$, Hye-Kyung SEO ${ }^{2}$, \\ Jung-Eun BAEK ${ }^{2}$, Jung-Wan KOO$^{1}$ and Hyunwook $\mathrm{KIM}^{2} *$ \\ ${ }^{1}$ Department of Occupational and Environmental Medicine, Seoul St. Mary's Hospital, College of Medicine, \\ The Catholic University of Korea, Republic of Korea \\ ${ }^{2}$ Department of Preventive Medicine, College of Medicine, The Catholic University of Korea, Republic of Korea \\ Received April 10, 2015 and accepted September 14, 2015 \\ Published online in J-STAGE November 3, 2015
}

\begin{abstract}
Tuberculosis infection is prevalent in Korea and health care workers are vulnerable to tuberculosis infection in the hospital. The aims of this study were to develop and validate an education program that teaches senior medical students how to wear and choose the proper size and type of respiratory protective equipment (RPE), which may help reduce the risk of contracting Mycobacterium tuberculosis (MTB) from patients. Overall, 50 senior medical students participated in this education program. Methods of choosing the proper type of RPE, performing a fit check of the RPE, and choosing a suitable mask size were taught by certified instructors using the real-time quantitative fit test (QNFT). The validity of education program was evaluated with qualitative fit test (QLFT) before and after the education as pass or fail. The education program was effective, as shown by the significantly pass rate (increased 30 to $74 \%$ ) in the QLFT after the education program $(p<0.05)$. Among study participants, changing mask size from medium to small significantly increased the pass rate $(p<0.001)$. Incorporation of this program into the medical school curriculum may help reduce risk of MTB infection in medical students working in the hospital.
\end{abstract}

Key words: Education, Medical student, Respiratory protective device, Fit test, Tuberculosis, Industrial hygiene

\section{Introduction}

Korea is at intermediate risk for Mycobacterium tuberculosiss (MTB) infection, with a prevalence and incidence of 89.6 and 71.4 , respectively, per 100,000 in $2013^{1)}$. MTB infection is also a major public health problem for health care workers (HCWs) in $\mathrm{Korea}^{2}$. According to a previous

*To whom correspondence should be addressed.

E-mail: hwkim@catholic.ac.kr

(C)2016 National Institute of Occupational Safety and Health study, MTB infection accounted for $19.5 \%$ of work-related infectious diseases in Korea from 2006 to $2011^{3)}$. Additionally, the majority of HCWs (70.6\%) considered MTB infection to be a serious health problem. Thus, programs for preventing MTB in HCWs are needed.

Respiratory protective equipment (RPE) is recommended for HCWs to prevent the spread of MTB and viral infections such as viral influenza ${ }^{4}$. HCWs in Korea, the increased incidence of work-related infectious disease was shown ${ }^{3}$. Several countries have already promulgated regular fit tests for HCWs working in the hospital to reduce incidence of infection from patients. Although every HCW 
should undergo fit tests for the RPE they use ${ }^{5-7)}$, these tests have not been used in Korea.

Like HCWs, medical students are at risk for MTB infection while they are taking clinical courses in the hospital ${ }^{8)}$. In Korea, senior medical students complete clinical training courses during a clerkship in the training hospital, and these individuals will also be clinicians as interns or residents in the near future. Therefore, educating medical students on the proper usage of RPE is essential because they are subject to MTB exposure in the hospital. However, no regular education program that teaches appropriate RPE usage exists for medical students in Korea.

The aims of this study were to develop and validate an education program for medical students that teaches the appropriate methods for wearing and choosing the proper size and type of RPE.

\section{Subjects and Methods}

\section{Study design and participants}

This analysis included the RPE education program for senior medical students in the Medical College at the Catholic University of Korea who had just finished the poly-clinical rotations periods. The education program was performed from July 2014 to September 2014. The quantitative fit test (QNFT) was used to educate the medical students, and the qualitative fit test (QLFT) was performed to analyze the rate of pass/fail before and after the RPE education program. In the St. Mary's hospitals, the disposable RPEs have been used, therefore, only disposable RPEs were verified by fit test. The sample size was calculated to estimate an ideal number of participants for this education program. A sample size of 50 participants was estimated to have sufficient power $(80 \%)$ to determine a true success rate of $74 \%(n=37)$ or $76 \%(n=38)$, assuming a $5 \%$ chance of a type I error and a $20 \%$ chance of a type II error ${ }^{9}$. Therefore, a total of 50 senior medical students volunteered to participate in the present study. The informed consent was obtained from participants. The study design, sampling methods, statistics, and practice were approved by the Institutional Review Board of the College of Medicine at The Catholic University of Korea (approval ID: MC14OISI0065).

\section{Qualitative fit test (QLFT)}

A QLFT was performed using a 3M Qualitative FitTesting tool (3M $\mathrm{M}^{\mathrm{TM}}-\mathrm{FT} 10$ kit, 3M Cp., St Paul, MN, USA). Before the fit test, a screening test was performed using a sensitivity solution $(0.83 \mathrm{~g}$ of saccharin $/ 100 \mathrm{ml}$ of distilled water). All participants passed the test. After the screening test, the participant drank 1 cup of water, gargled his or her mouth with 2 cups of water, and left the experiment room. The participant selected and donned the RPE. Procedures of the fit test consisted of normal breathing, deep breathing, moving the head side to side, moving the head up and down, talking loudly, and a second bout of normal breathing. The test solution was 100 times more concentrated ( $83 \mathrm{~g}$ of saccharin $/ 100 \mathrm{ml}$ of distilled water) than the sensitivity solution. The QLFT was performed before and after the RPE education program.

\section{Quantitative fit test (QNFT)}

The Portacount Pro Plus 8038 (TSI Inc., Shoreview, MN, USA) was used for the QNFT. This instrument was capable of supporting the QNFT for N95 masks. The probe for QNFT was installed at the midpoint between the nose and mouth. The QNFT procedures were similar to those of the QLFT, with the addition of grimace, bend, and toe-touch movements. During the QNFT, participants were able to monitor the changes in fit factors in real time.

All the processes for QNFT and QLFT were performed according to OSHA (29 CFR 1910.134) standards ${ }^{5)}$.

\section{Quality control of QLFT and QNFT}

All practice and quality control processes were reviewed by two occupational physicians and an industrial hygienist. Two occupational physicians (BJS and MJP), who were certified by the $3 \mathrm{M}$ company to administer the FIT TEST (QLFT and QNFT) on June 20th 2014, performed the fit test.

\section{Statistical analysis}

All statistical analyses were performed using SAS 9.3 (SAS Institute, Cary, NC, USA). The characteristics of participants were described as means and standard deviation (SD) for continuous variables, and frequency and proportion (\%) for categorical variables, according to final fit test results. Student's $t$-tests and $\chi^{2}$ tests were used to evaluate differences in continuous variables (such as age and anthropometrical indices) and categorical variables, respectively, according to final fit test results. To validate the RPE education program, the program should satisfy two requisitions. First, the number of passed participants for final QLFT is up to 37 (74\%) or more will be considered as effective and valid education and practice program. The McNemar test was used to compare the proportion of individuals who passed the QLFT before and after the program with QNFT. Second, the statistical significant changes at the NcNemar test for QLFT should be shown before and 
after the education program with QNFT. A $p$-value less than 0.05 was considered to be statistically significant.

\section{Results}

\section{Education and practice program development}

The scheme of the education and practice program for RPE is described in Fig. 1. The program consist of the pre-evaluation with QLFT (pass/fail), the education and practice program, and the post-evaluation with QLFT (pass/ fail). The QLFT was used to estimate the effectiveness of education program. The QNFT was used to educating the medical students. The education and practice program consisted of three domains; choosing the proper types of RPE, wearing RPE (which included a fit check), and choosing a suitable mask size. All participants have donned four different respirators: VFlex 9102, VFlex 9102S, and 3M 1870 (3M Cp., St Paul, MN, USA), and procedure mask 82001 (YuHan-Kimberly, Seoul, South Korea) during the QNFT. Wearing and fitting the different sizes of VFLEX 9102/9102S RPEs and monitoring the real-time fit factors of participants were designed to find the proper RPE size for individual fitting. Passing the QNFT was regarded as achieving a minimum fit factor of 100 . Comparing the real-time fit factors between the N95 RPE (3M 1870) and procedure mask (surgical mask 82001) consisted of finding the appropriate mask type after exposure to the tuberculosis droplet nuclei $(1-5 \mu \mathrm{m})$. Students were instructed on wearing and fit checks with instruction figures (supplement 1), and were rechecked when participants switched to other RPE. Figure 2 summarized the process of education and practice program for respiratory protection equipments.

\section{Program effectiveness evaluation}

Table 1 shows the general characteristics and anthropo-

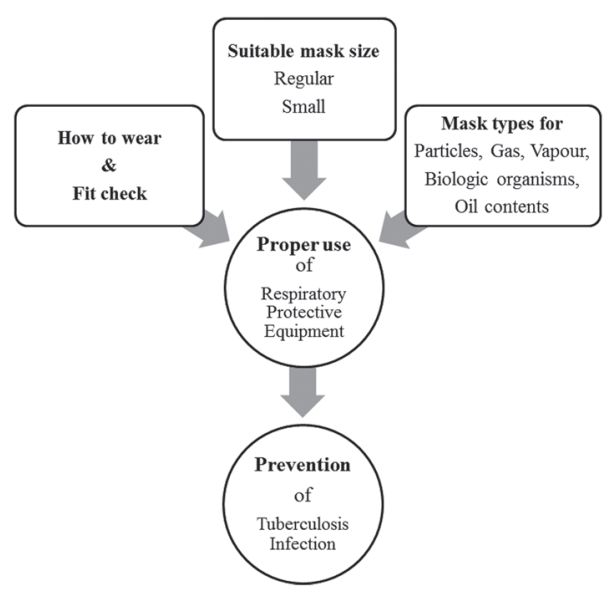

Fig. 1. Structural assessment of successful respiratory protective equipment use.

metrical results according to the final fit test. The mean (SD) age of the participants was 27.0 (1.7) yr. The mean (SD) lip and face lengths were $39.7(3.8) \mathrm{cm}$ and 116.8 (7.0) $\mathrm{cm}$, respectively. The pass rate of the final fit test was not different between the genders $(p>0.05)$. The proportion of individuals who passed the QLFT for RPE was 30\% $(\mathrm{n}=15)$ before the program and $74.0 \%(n=37)$ after the program.

The QLFT results before and after the RPE practice program are summarized in Table 2. A total of $24(68.6 \%)$ participants who failed the QLFT before the program passed after the practice program. Immediate effectiveness of the education program was statistically significant $(p<0.001)$. Subgroup analysis according to mask size showed that the education program was significantly more effective in those who changed from the medium to the small size between the two tests $(p<0.01)$. For those who used same size of RPEs at before and after the education and practice program (medium to medium and small to

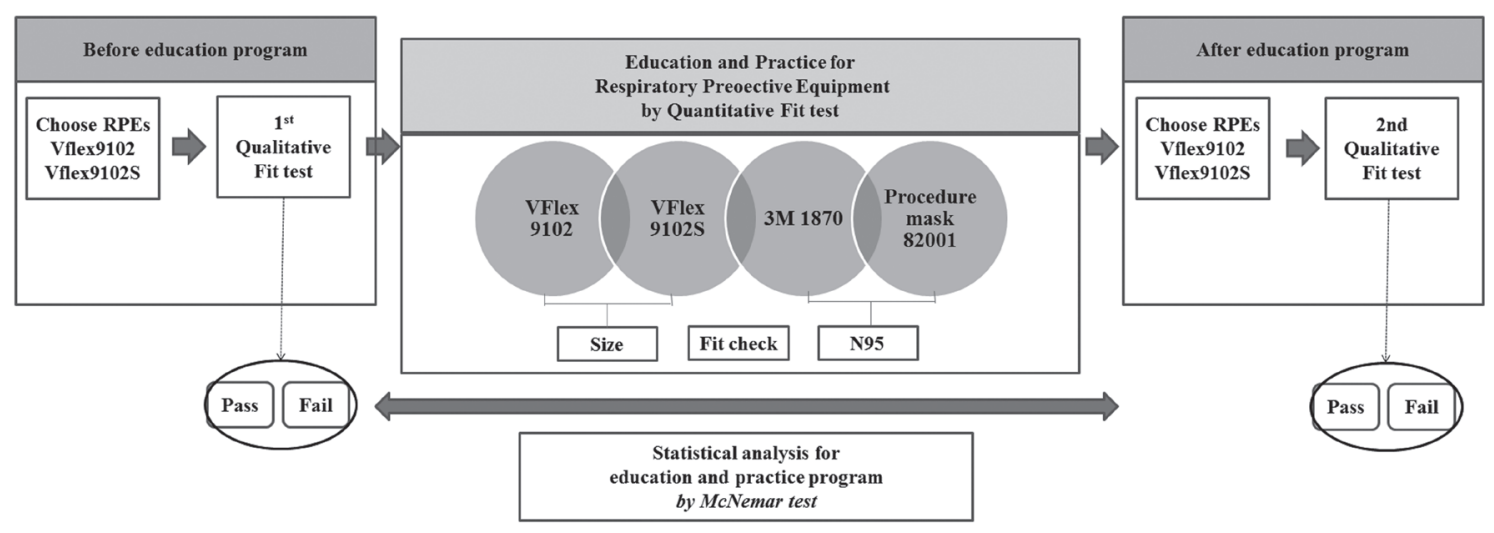

Fig. 2 Scheme of education and practice program for respiratory protection equipments. 
Table 1. Distribution of general characteristics, anthropometrical index, and mask change in study participants according to final fit test results

\begin{tabular}{|c|c|c|c|c|c|}
\hline & \multicolumn{4}{|c|}{ Final qualitative fit test results for RPE } & \multirow{3}{*}{$p$-value } \\
\hline & \multicolumn{2}{|c|}{ Pass } & \multicolumn{2}{|c|}{ Fail } & \\
\hline & $\mathrm{N}$ (mean) & $\%(\mathrm{SD})$ & $\mathrm{N}$ (mean) & $\%(\mathrm{SD})$ & \\
\hline Age & $(27.2)$ & $(1.6)$ & $(27.3)$ & (1.9) & 0.789 \\
\hline Gender & & & & & 1.000 \\
\hline Female & 14 & 37.8 & 5 & 38.5 & \\
\hline Male & 23 & 62.2 & 8 & 61.5 & \\
\hline \multicolumn{6}{|l|}{ Anthropometrical index } \\
\hline Lip length (cm) & $(39.8)$ & $(4.1)$ & $(39.1)$ & $(2.8)$ & 0.557 \\
\hline Face length $(\mathrm{cm})$ & $(117.0)$ & (7.4) & $(116.2)$ & $(5.6)$ & 0.754 \\
\hline \multicolumn{5}{|c|}{ Mask size change (pre to post education) } & 0.629 \\
\hline Small $^{*}$ to small ${ }^{*}$ & 12 & 32.4 & 3 & 23.1 & \\
\hline Small $^{*}$ to medium ${ }^{\dagger}$ & 2 & 5.4 & 2 & 15.4 & \\
\hline Medium $^{\dagger}$ to small* & 12 & 32.4 & 5 & 38.4 & \\
\hline Medium $^{\dagger}$ to medium ${ }^{\dagger}$ & 11 & 29.8 & 3 & 23.1 & \\
\hline Total & 37 & 100.0 & 13 & 100.0 & \\
\hline
\end{tabular}

RPE: respiratory protective equipment

*VFlex 9102S, †VFlex 9102

Table 2. Immediate effectiveness of the RPE education program

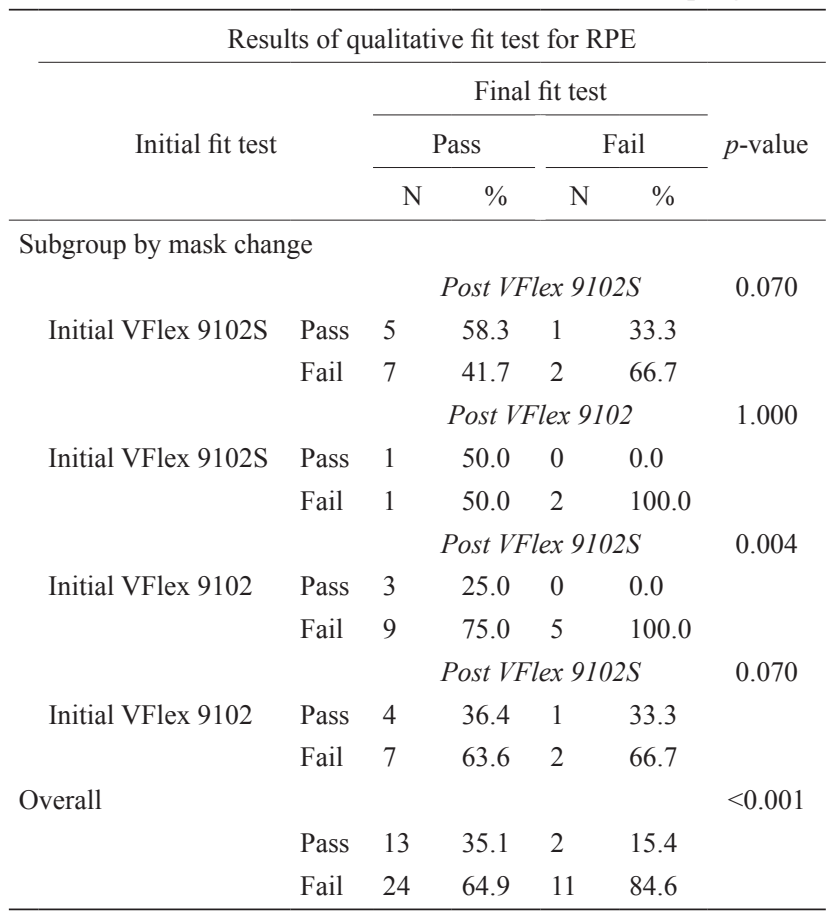

RPE: respiratory protective equipment

Statistical analysis: McNemar test

small), over the seventy seven percent $(n=14)$ of them was pass after the program.

\section{Discussion}

In the present study, a newly introduced education program that instructed on the proper use of RPE significantly increased the pass rate of the QLFT of senior medical students in a medical college in Korea.

Choosing the proper type of RPE is the most important factor to ensure adequate protection. Currently, procedure or simple surgical masks are commonly used by HCWs. However, previous studies indicate that surgical masks are not adequate for preventing $\mathrm{MTB}^{10-13)}$. Although the filter for fluid resistance and bacterial filtration efficiency is confirmed by the manufacturers, the facial fit and protection factors are not certified or guaranteed ${ }^{13)}$. Leakages can occur through the gaps between the face and mask. Therefore, comparing real-time fit factors between procedure masks or simple surgical masks and N95 RPE was one of the education points to inform the medical students of the proper RPE type to prevent MTB infection.

Fit check is important to prevent leakage ${ }^{14)}$. In the present study, participants wore three RPE types and one procedure mask, and performed fit checks and tests four times for each device. The repeated checks and tests may increase the pass rate by providing feedback on necessary adjustments. Ciotti et al. found that those who performed the fit test three times on RPEs had a successful fit test of $60-90 \%$, whereas $0-60 \%$ of those who tested only one mask had a successful fit test ${ }^{15)}$. However, Ciotti et al. 
emphasized that this approach will only achieve a rate of $100 \%$ if the RPE is adapted correctly to the facial anthropometry of the participant ${ }^{15}$.

Most commercial RPE is made according to the facial anthropometrical standards of individuals in US or European countries. Due to the racial differences between Koreans and westerners, medium sizes of RPE may be too large or have a poor fit for Korean individuals. Therefore, several previous studies have aimed to measure and find the proper size of RPE for Korean anthropometrical dimensions ${ }^{16-18)}$. Unfortunately, we were unable to find the N95 RPE for hospital use that was available in both medium and small sizes. Thus, the VFlex 9102 and $9102 \mathrm{~S}$ were selected to teach the proper size of RPE to use in this study. The small- and medium-size RPE was a sister model of N95 RPE (3M VFlex 9105), which was the same product but was available under a different model number in a different country (which was confirmed by the $3 \mathrm{M}$ manufacturer). After wearing two different sizes in the real-time QNFT, participants likely learned which size fit them most appropriately, which was indicated by the increase in QLFT pass rates after education. Over sixty percent of individuals ( $\mathrm{n}=9$ of 14 ) who failed the test before the program with the medium size passed the test after the program, suggesting that the small size based on Western facial anthropometry may be a better fit for the Korean participants. However, the present study was focused on the effectiveness of education for appropriate RPE fitting and not to verify the proper sizing of RPE for Koreans. Thus, further evaluation of proper RPE size in a Korean population should be performed.

The minimum fit factor for a successful fit test was set at 100 in the present study based on previous research ${ }^{5)}$. After QNFT, the RPE size that was successfully fit tested (small or medium size) was recommended as the proper size. Previous studies have shown discordance between fit test results and the workplace protection factor ${ }^{19,20)}$. Thus, the results of the fit test in the present study do not translate to a perfect protection from MTB or other hazardous materials in the real setting, which may be a significant limitation for adopting this education program in the real setting. However, several studies have estimated a significant correlation between the fit test results and protection factor in the workplace $\left(\mathrm{R}^{2}=0.38-0.55\right)^{20,21)}$. According to US government documents for RPE, an assigned protection factor of 10 was recommended to indicate RPE safety, and the fit factor should be over 100 to satisfy this level ${ }^{5}$. Even with this guideline, participants failed the fit test even when abiding by the recommendations of the QNFT.
Follow-up evaluation is warranted to reinforce the applicability of this RPE education program to HCW safety.

Our study has several limitations. First, the QLFT was used to verify the effectiveness of the education program for RPE. The QLFT with saccharin is a subjective test that depends on the ability of the participant to taste sweetness $^{22)}$, and those who have deficits in olfactory senses were unable to complete the QLFT. Therefore, we used the sensitivity test with diluted saccharin before the QLFT to ensure that the participants were capable of tasting the saccharin in the QLFT. All of our participants passed the sensitivity test, and instructors were certified to administer QLFT before this study. Second, although there were two QLFTs (a sweet kit with saccharin and a bitter kit with denatonium benzoate), we only used the QLFT with saccharin in this study due to a previous report of adverse effects associated with denatonium benzoate ${ }^{23)}$. Third, this study was designed to validate the effectiveness of the newly developed education program for RPE, and therefore the predictive factors for failing the fit test were not assessed. Further evaluation and measures should be performed to protect those who failed the fit test. Fourth, the QNFT was not used to pre-post education program. While the QNFT the count numbers of particle in/out side of RPEs were shown in the monitor. To reduce the student's

The present study had several strengths. Previous studies have aimed to validate specific components such as effectiveness of RPE type, size, or fit check ${ }^{15,24,25)}$. Those components are important to protect users, and the RPE would be useless if one of those components was not satisfied. Therefore, actual practices in the present study consisted of choosing proper types of RPE, wearing RPE (including a fit check), and choosing a suitable mask size to determine RPE fit. Furthermore, we controlled for potential confounders such as cigarette smoking, eating, and shaving facial hair before the fit test.

In conclusion, the present education program for RPE use for medical students was effective, as shown by the increase in the pass rate of the QLFT after taking this course. Thus, including this program in the regular curriculum of medical schools may help to reduce the risk of MTB infection in medical students working in the hospital. Moreover, further studies on possible countermeasures for those who failed the fit test should be performed.

\section{Acknowledgements}

The authors acknowledge Dr. Bum-Seok Cho for being a model of fit check in the figure. The authors wish to 
acknowledge the financial support of the Catholic Medical Center Research Foundation received in the 2014 program year.

\section{References}

1) Korea Center for Disease Control and Prevention Annual report on the notified tuberculosis in Korea, 2013. http:// tbfree.cdc.go.kr/tbfree/cmm/BoardView.do?boardType=RE PORT\&no $=402 \&$ id $=4500 \&$ page $2=1 \&$ pageType $=$. Accessed April 1, 2015.

2) Kim EA, Kang SK (2013) Historical review of the list of occupational diseases recommended by the International Labour organization (ILO). Ann Occup Environ Med 25, 14. [Medline] [CrossRef]

3) Myong JP, Ahn YS, Kim HR, Kim YJ, Park CY, Koo JW (2013) Work-related infectious diseases among Korean workers compensated under the Industrial Accident Compensation Insurance Law, 2006-2011. Int J Occup Environ Health 19, 344-51. [Medline] [CrossRef]

4) Jensen PA, Lambert LA, Iademarco MF, Ridzon R, CDC (2005) Guidelines for preventing the transmission of Mycobacterium tuberculosis in health-care settings, 2005. MMWR Recomm Rep 54 RR-17, 1-141. [Medline]

5) United States Department of Labor, Occupational Safety and Health Administration Respiratory Protection (Regulations (Standards-29 CFR), Standard Number: 1910.134). https:// www.osha.gov/pls/oshaweb/owadisp.show_document?p table $=$ standards\&p_id=12716. Accessed September 16, 2015.

6) Notarianni GL (1993) Recommendations for TB respiratory protection. J Healthc Mater Manage 11, 28-32. [Medline]

7) Australian Government Department of Health and AgeingInterim National Pandemic Influenza Clinical Guidelines. http://www.public.health.wa.gov.au/cproot/2246/2/ pandemic-clinical-gl.pdf. Accessed April 1, 2015.

8) Teixeira EG, Menzies D, Comstock GW, Cunha AJ, Kritski AL, Soares LC, Bethlem E, Zanetti G, RuffinoNetto A, Belo MT, Selig L, Branco MM, Cherri D, Maia S, Marandino R, Luiz RR, Chaisson RE, Trajman A (2005) Latent tuberculosis infection among undergraduate medical students in Rio de Janeiro State, Brazil. Int J Tuberc Lung Dis 9, 841-7. [Medline]

9) Landsittel D, Zhuang Z, Newcomb W, Berry Ann R (2014) Determining sample size and a passing criterion for respirator fit-test panels. J Occup Environ Hyg 11, 77-84. [Medline] [CrossRef]

10) Grinshpun SA, Haruta H, Eninger RM, Reponen $T$, McKay RT, Lee SA (2009) Performance of an N95 filtering facepiece particulate respirator and a surgical mask during human breathing: two pathways for particle penetration. J Occup Environ Hyg 6, 593-603. [Medline] [CrossRef]

11) Lee SA, Grinshpun SA, Reponen $T$ (2008) Respiratory performance offered by N95 respirators and surgical masks: human subject evaluation with $\mathrm{NaCl}$ aerosol representing bacterial and viral particle size range. Ann Occup Hyg 52, 177-85. [Medline] [CrossRef]

12) Naidoo S, Mahommed A (2002) Knowledge, attitudes, behaviour and prevalence of TB infection among dentists in the western Cape. SADJ 57, 476-8. [Medline]

13) Oberg T, Brosseau LM (2008) Surgical mask filter and fit performance. Am J Infect Control 36, 276-82. [Medline] [CrossRef]

14) Danyluk Q, Hon CY, Neudorf M, Yassi A, Bryce E, Janssen B, Astrakianakis G (2011) Health care workers and respiratory protection: is the user seal check a surrogate for respirator fit-testing? J Occup Environ Hyg 8, 267-70. [Medline] [CrossRef]

15) Ciotti C, Pellissier G, Rabaud C, Lucet JC, Abiteboul D, Bouvet E, GERES (2012) Effectiveness of respirator masks for healthcare workers, in France. Med Mal Infect 42, 264-9. [Medline] [CrossRef]

16) Han DH, Choi KL (2003) Facial dimensions and predictors of fit for half-mask respirators in Koreans. AIHA J (Fairfax, Va) 64, 815-22. [Medline] [CrossRef]

17) Han DH, Rhi J, Lee J (2004) Development of prototypes of half-mask facepieces for Koreans using the 3D digitizing design method: a pilot study. Ann Occup Hyg 48, 707-14. [Medline] [CrossRef]

18) Kim H, Han DH, Roh YM, Kim K, Park YG (2003) Facial anthropometric dimensions of Koreans and their associations with fit of quarter-mask respirators. Ind Health 41, 8-18. [Medline] [CrossRef]

19) Duling MG, Lawrence RB, Slaven JE, Coffey CC (2007) Simulated workplace protection factors for half-facepiece respiratory protective devices. J Occup Environ Hyg 4, 420-31. [Medline] [CrossRef]

20) Zhuang Z, Coffey CC, Jensen PA, Campbell DL, Lawrence RB, Myers WR (2003) Correlation between quantitative fit factors and workplace protection factors measured in actual workplace environments at a steel foundry. AIHA J (Fairfax, Va) 64, 730-8. [Medline] [CrossRef]

21) Han DH (2002) Correlations between workplace protection factors and fit factors for filtering facepieces in the welding workplace. Ind Health 40, 328-34. [Medline] [CrossRef]

22) Coffey CC, Lawrence RB, Zhuang Z, Campbell DL, Jensen PA, Myers WR (2002) Comparison of five methods for fittesting N95 filtering-facepiece respirators. Appl Occup Environ Hyg 17, 723-30. [Medline] [CrossRef]

23) Youakim S (2007) Adverse reactions associated with respirator fit testing of healthcare workers in British Columbia, Canada: a review of compensation claim cases. Arch Environ Occup Health 62, 197-200. [Medline] [CrossRef]

24) Benson SM, Novak DA, Ogg MJ (2013) Proper use of surgical $n 95$ respirators and surgical masks in the OR. AORN J 97, 457-67, quiz 468-70. [Medline] [CrossRef]

25) Coffey CC, Lawrence RB, Campbell DL, Zhuang Z, Calvert CA, Jensen PA (2004) Fitting characteristics of eighteen N95 filtering-facepiece respirators. J Occup Environ Hyg 1, 262-71. [Medline] [CrossRef] 